International Journal of Pure and Applied Mathematics

Volume 84 No. 2 2013, 1-13

ISSN: 1311-8080 (printed version); ISSN: 1314-3395 (on-line version)

url: http://www.ijpam.eu

doi: http://dx.doi.org/10.12732/ijpam.v84i2.1

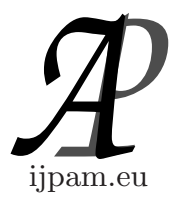

\title{
FRACTIONAL DIFFERENTIATION MATRICES FOR SOLVING FRACTIONAL ORDERS DIFFERENTIAL EQUATIONS
}

\author{
M. El-Kady ${ }^{1}$, Amaal El-Sayed ${ }^{2}$ \\ ${ }^{1,2}$ Department of Mathematics \\ Faculty of Science \\ Helwan University, Cairo, EGYPT
}

\begin{abstract}
This paper gives a new formula for the fractional differentiation matrix based on shifted Chebyshev polynomials for solving fractional order ordinary differential equations (FODEs). In addition, we will estimate the error bound of the in the largest element of that matrix. Some numerical examples are included to confirm the accuracy of the new formula and illustrate the practical usefulness of our method.
\end{abstract}

AMS Subject Classification: 65G20, 65L70, 65L09

Key Words: fractional differential equations, shifted Chebyshev polynomials, differentiation matrices

\section{Introduction}

The fractional differential equations are posing many problems in engineering and physics. The subject of fractional calculus and its applications has gained considerable popularity and importance during the past three decades or so, due mainly to its demonstrated applications in numerous seemingly diverse and widespread fields of science and engineering. These problems have a large number of applications in many different fields, e.g., mechanical system [3], automotive vehicle design [11] and manufacturing process [8]. Some of the

Received: March 26, 2012

(c) 2013 Academic Publications, Ltd. url: www.acadpubl.eu 
areas of present-day applications of fractional models include fluid flow, solute transport or dynamical processes in self-similar, electromagnetic theory and control theory of dynamical systems.

Recently, fractional differential equations are generalizations of ordinary differential equations to an arbitrary (non integer) order. Due to the

extensive applications of FDEs in engineering and science, research in this area has grown significantly all around the world. Several authors have studied spectral differentiation matrices, mainly for Chebyshev differentiation matrices $[5,6,9]$. In many applications, the formulae of spectral differentiation matrix are appeared as a relation between the expansion coefficients of derivatives with those of the function itself. Clearly, formulae of the expansion coefficients of a general order derivative of finite differentiable orthogonal polynomials in terms of those polynomials themselves are available for Chebyshev [8]. These naive algorithms for computing pseudospectral differentiation matrices suffer from severe loss of accuracy due to round off errors.

On the other hand, Chebyshev polynomials have proven successfully in the numerical solution of various boundary value problems [2]. Chebyshev polynomials are important in approximation theory because the roots of the Chebyshev polynomials are used as nodes in polynomial interpolation. The resulting interpolation polynomial minimizes the problem of Runge's phenomenon and provides an approximation that is close to the polynomial of best approximation to a continuous function under the maximum norm. This approximation leads directly to the method of Clenshaw-Curtis quadrature [1].

This paper aims to formulate the fractional differentiation matrix based on shifted Chebyshev polynomials and it may use for solving fractional order ordinary differential equations (FODEs).

The organization of this paper is as follows: In Section 2, basic formulation of the shifted Chebyshev polynomials are presented. In Section 3, fractional Chebyshev differentiation matrices are presented. We analyzed the error upper bounds of the method in Section 4. In Section 5 the proposed matrices are applied to several examples. Also a conclusion is given in Section 6

\section{Preliminaries and Fractional Derivatives}

Shifted Chebyshev polynomials of the first kind $T_{n}^{*}(X)$ are defined through the identity:

$$
T_{n}^{*}(X)=\cos (2 n \theta)
$$


where $x=\cos ^{2} \theta$ and $0 \leq x \leq 1$

The recurrence formula is as follows:

$$
T_{n}^{*}(X)=2(2 x-1) T_{n-1}^{*}(x)-T_{n-2}^{*}(x), n=2,3, \ldots
$$

with the initial conditions:

$$
T_{0}^{*}(x)=1, t_{1}^{*}(x)=2 x-1
$$

The shifted Chebyshev polynomials $T_{n}^{*}(X)$ can be expanded in power series:

$$
T_{n}^{*}(X)=n \sum_{k=0}^{n}(-1)^{n-k} \frac{(2)^{2 k}(n+k-1) !(x)^{k}}{(2 k) !(n-k) !}, n>0
$$

The orthogonality property of the shifted Chebyshev polynomials of the first kind is as follows:

$$
\int_{0}^{1} T_{i}^{*}(x) T_{j}^{*}(x) w(x) d x= \begin{cases}0 & i \neq j \\ \pi & i=j=0 \quad, 0 \leq i, j \leq N \\ \frac{\pi}{2} & i=j \neq 0\end{cases}
$$

we observe that the shifted Chebyshev polynomials form an orthogonal set on the interval $0 \leq x \leq 1$ with the weighting function $w(x)=\left(x-x^{2}\right)^{-1 / 2}$.

One of the major advantages of using shifted Chebyshev polynomials $T_{n}(x)$ as expansion functions is the good representation of smooth functions by finite Chebyshev expansions. Also, the shifted Chebyshev expansion coefficients $a_{n}$ approach zero faster than any inverse power in $n$ as $n \rightarrow \infty$ [3].

There are many quadratures of shifted Chebyshev polynomials such as:

1. Shifted Chebyshev zero points for $T_{n}^{*}(x)$ :

$$
x_{i}=\frac{1}{2}\left(\cos \left(\frac{\pi(2 i+1)}{2 N}\right)+1\right) \text { for } 1 \leq i \leq N .
$$

2. Shifted Chebyshev extreme points for $T_{n}^{*}(x)$ :

$$
x_{i}=\frac{1}{2}\left(\cos \left(\frac{i \pi}{N}\right)+1\right) 0 \leq i \leq N .
$$

Fractional derivatives:

Let $\alpha \in \mathbb{R}_{+}$and $n=[\alpha]$. The operator $D^{\alpha}$ is defined by:

$$
D^{\alpha} f(x)=\frac{1}{\Gamma(n-\alpha)} \int_{0}^{x}(x-t)^{n-\alpha-1}\left(\frac{d}{d x}\right)^{n} f(t) d t
$$


for $n-1<\alpha \leq n, n \in N$, is called the Caputo differential operator of order $\alpha$. We are ready now to define a real-indexed derivative with $D^{\alpha}$ with $\alpha \in \mathcal{R}$, of a monomial, as

$$
D^{\alpha}\left(x^{\beta}\right)=\frac{\Gamma(\beta+1)}{\Gamma(\beta+1-\alpha)} x^{\beta-\alpha}
$$

One can notice that,

$$
D^{\alpha}(c)=0
$$

where $c$ is constant. There are some properties of fractional derivatives such as:

a) The first property of interest is that of association

$$
D^{\alpha}(c f(x))=c D^{\alpha}(f(x)) .
$$

b) The second property we would like to introduce into the fractional calculus is the distributive law

$$
D^{\alpha}(f(x) \pm g(x))=D^{\alpha}(f(x)) \pm D^{\alpha}(g(x)) .
$$

c) The final property is that the operator obeys Leibniz rule for taking the derivative of the product of two functions

$$
D^{\alpha}(f(x) g(x))=\sum_{k=0}^{\alpha}\left(\begin{array}{c}
\alpha \\
k
\end{array}\right) D^{\alpha-k}(g(x)) D^{k}(f(x))
$$

Fractional derivatives possess a memory effect. This can be very useful in modeling behavior, but can also be computationally expensive. Any numerical approximation of a fractional differential equation requires an exponential increase in work with increasing time. If multiple instances of fractional derivatives are included the workload again increases exponentially.

\section{Fractional Shifted Chebyshev Differentiation Matrices}

The basic idea of pseudospectral approximations method is to assume the unknown $y(x)$ of the differential equation can be approximated by sum of $N+1$ basis functions $\phi_{n}(x)$ as follows:

$$
y_{n}(x) \sum_{n=0}^{N} a_{n} \phi_{n}(x)
$$

This series is substituted into the differential equation:

$$
L^{(\alpha)} y=f(x), 0 \leq x \leq 1
$$


where $L^{(\alpha)}$ is a fractional differential operator and $f(x)$ is a given function. The result is called residual function and defined by:

$$
R\left(x, a_{0}, a_{1}, a_{2}, \ldots, a_{N}\right)=L y_{N}-f
$$

This is identically equal to zero for the exact solution. In this paper, the choice of $\phi_{n}(x)$ is shifted Chebyshev polynomials which are taken with their associated Gauss Lobatto points (GLP) in the interval $[0,1]$. The set of collocation points is related to the set of basis function as the nodes of quadrature formulae which are used in the computation of the spectral coefficients from the grid values. Consider the function $f(x)$ can be approximated in the interval $[0,1]$ by using shifted Chebyshev polynomials $T_{n}^{*}(x)$ as follows [1]:

$$
f(x)=\sum_{n=0}^{N} \theta_{n} a_{n} T_{n}^{*}(x)
$$

where

$$
a_{n}=\frac{2}{N} \sum_{j=0}^{N} \theta_{j} f\left(x_{j}\right) T_{n}^{*}\left(x_{j}\right)
$$

for $\theta_{n}, \theta_{j}$ are equal to 1 for all $n, j=1,2, \ldots, N-1$, and $\theta_{0}=\theta_{N}=\frac{1}{2}$. The fractional derivative $f^{(\alpha)}(x)$ at the extreme points $x_{i}$ is given by:

$$
f^{(\alpha)}\left(x_{i}\right)=\sum_{n=0}^{N} \theta_{n} a_{n} T_{n}^{*(\alpha)}\left(x_{i}\right)
$$

Chebyshev polynomials as follows:

$$
T_{n}^{*(\alpha)}(x)=n \sum_{k \geq\lceil\alpha\rceil}^{n}(-1)^{n-k} \frac{(2)^{2 k}(n+k-1) ! \Gamma(k+1)(x)^{k-\alpha}}{(2 k) !(n-k) ! \Gamma(k+1-\alpha)}
$$

where $k>0, n=\lceil\alpha\rceil,\lceil\alpha\rceil+1, \ldots, N$, and

$$
T_{n}^{*(\alpha)}(x)=0, \quad n=0,1, \ldots,\lceil\alpha\rceil-1
$$

The meaning of $\lceil\alpha\rceil$ is the ceiling function which denotes to the smallest integer greater than or equal to $\alpha$. The expression of fractional differentiation matrix can be formulated as follows:

$$
f^{(\alpha)}\left(x_{i}\right)=\sum_{j=0}^{N} d_{i, j}^{(\alpha)} f\left(x_{j}\right)
$$


where

$$
d_{i, j}^{(\alpha)}=\frac{2 \theta_{j}}{N} \sum_{n=0}^{N} \theta_{n} T_{n}^{*}\left(x_{j}\right) T_{n}^{* \alpha}\left(x_{i}\right)
$$

The matrix form is:

$$
\mathbf{f}^{(\alpha)}=\mathbf{D}^{(\alpha)} \mathbf{f}
$$

The elements of the fractional differentiation matrix $\mathbf{D}^{(\alpha)}$ are:

$$
d_{i, j}^{(\alpha)}=\frac{2}{N}\left[\begin{array}{cccc}
0 & 0 & \cdots & 0 \\
\frac{1}{2} \mathbf{A}_{1,0}^{(\alpha)} & \mathbf{A}_{1,1}^{(\alpha)} & \cdots & \frac{1}{2} \cdot \mathbf{A}_{1, N}^{(\alpha)} \\
\vdots & \vdots & \ddots & \vdots \\
\frac{1}{2} \mathbf{A}_{N, 0}^{(\alpha)} & \mathbf{A}_{N, 1}^{(\alpha)} & \cdots & \frac{1}{2} \mathbf{A}_{N, N}^{(\alpha)}
\end{array}\right]
$$

where,

$$
\mathbf{A}_{i, j}^{(\alpha)}=\sum_{n=0}^{N} \theta_{n} T_{n}^{*}\left(x_{j}\right) T_{n}^{*(\alpha)}\left(x_{i}\right)
$$

In case of $\alpha=0.5$ and $N=4$, the elements of the fractional differentiation matrix are as follows:

$$
d_{i, j}^{(0.5)}=\left[\begin{array}{ccccc}
0 & 0 & 0 & 0 & 0 \\
-2.4362 & 2.1302 & 0.4392 & -0.2286 & 0.0954 \\
-0.5395 & -1.2067 & 1.3678 & 0.5987 & -0.2204 \\
-0.7706 & 0.2343 & -1.2986 & 1.2114 & 0.6235 \\
-0.4621 & -0.3568 & 0.1290 & -2.9101 & 3.6001
\end{array}\right]
$$

\section{Error Analysis}

In this section the error effects of the largest element of the fractional differentiation matrix in case of integer and fractional numbers is presented. Also, two numerical tests for integer derivative and fractional derivative are given in double precision with unit round off $\varepsilon=2.22 \times 10^{-16}$. All the programs have been written in Matlab 10.

\subsection{Error Bound for the First Order Derivatives}

We investigate the effect of round off error in the matrix $\mathbf{D}^{(\alpha)}$ with $\alpha=1$ at shifted Chebyshev extreme points. In finite precision arithmetic, we have

$$
\tilde{x}_{1}=x_{1}+\delta
$$


where $\delta$ denotes a small error. In this section, we present the error $\tilde{d}_{01}^{(\alpha)}-d_{01}^{(\alpha)}$ at the shifted Chebyshev extreme points [9]:

$$
d_{01}^{(1)}=\frac{-2}{x_{0}-x_{1}}=\frac{-2}{\frac{\pi^{2}}{4 N^{2}}+O\left(\frac{1}{N^{4}}\right)}=\frac{-2}{\frac{\pi^{2}}{4 N^{2}}\left(1+O\left(\frac{1}{N^{2}}\right)\right)}=-\frac{8 N^{2}}{\pi^{2}}\left(1-O\left(\frac{1}{N^{2}}\right)\right) .
$$

The element $d_{0,1}^{(1)}$ is the major elements concerning its values. Accordingly, it bears the major error responsibility comparing the other elements. In Ref. [9], the authors computed the error as follows:

$$
\tilde{d}_{01}^{(1)}-d_{01}^{(1)}=\frac{32 N^{4}}{\pi^{4}}
$$

They found that the error is of order $O\left(N^{4} \delta\right)$, whereas the authors in [6] show that the error in the element $d_{0,1}^{(1)}$ is of order $O\left(N^{2} \delta\right)$. By using Eq. (14), we investigate here the effect of rounding for the element $\tilde{d}_{01}^{(1)}-d_{01}^{(1)}$, i.e.,

$$
d_{01}^{(1)}=\frac{2}{N} \sum_{n=0}^{N} 2(-1)^{2 n} \theta_{n}\left(2 x_{n}-1\right) n^{2}
$$

with the error bound

$$
\begin{aligned}
\tilde{d}_{01}^{(1)}-d_{01}^{(1)}= & \frac{8}{N} \sum_{n=0}^{N}(-1)^{2 n} \theta_{n} \delta_{n} \\
& \leq\left[\frac{8}{3} N^{2}+4 N+\text { frac } 43\right] \delta
\end{aligned}
$$

where, $\delta=\max _{0 \leq n \leq N}\left\{\delta_{n}\right\}$.

The efficiency of presented investigation is shown in Table (4.1).

Table (4.1): Computed error $\tilde{d}_{01}^{(1)}-d_{01}^{(1)}$

\begin{tabular}{|c|c|c|}
\hline $\mathrm{N}$ & Eq.(15) & Eq.(16) \\
\hline 8 & $2.9872 \mathrm{e}-013$ & $4.5288 \mathrm{e}-014$ \\
\hline 12 & $1.5123 \mathrm{e}-012$ & $9.6200 \mathrm{e}-014$ \\
\hline 16 & $4.7795 \mathrm{e}-012$ & $1.6606 \mathrm{e}-013$ \\
\hline 20 & $1.1669 \mathrm{e}-011$ & $2.5486 \mathrm{e}-013$ \\
\hline 24 & $2.4196 \mathrm{e}-011$ & $3.6260 \mathrm{e}-013$ \\
\hline 28 & $4.4827 \mathrm{e}-011$ & $4.8929 \mathrm{e}-013$ \\
\hline
\end{tabular}

On the other hand, the following example is computed for new formula (14) when $\alpha=1,2$ for the test function $f(x)=x^{8}$. The results are compared with the Chebyshev differentiation matrix in [7] for different numbers of GCL points as shown in Table (4.2). 
Table (4.2): Observed errors for first and second derivatives

\begin{tabular}{|c|c|c|c|c|}
\hline $\mathrm{N}$ & $\alpha=1[7]$ & $\alpha=1(E q .(14)$ & $\alpha=2[7]$ & $\alpha=2(E q .(14)$ \\
\hline 8 & $4.3656 \mathrm{e}-011$ & $3.0198 \mathrm{e}-014$ & $1.1642 \mathrm{e}-009$ & $4.2633 \mathrm{e}-014$ \\
\hline 12 & $1.0477 \mathrm{e}-009$ & $3.4106 \mathrm{e}-013$ & $6.7055 \mathrm{e}-008$ & $1.5461 \mathrm{e}-011$ \\
\hline 16 & $4.6566 \mathrm{e}-010$ & $4.5297 \mathrm{e}-014$ & $5.9605 \mathrm{e}-008$ & $7.2760 \mathrm{e}-012$ \\
\hline 20 & $5.2387 \mathrm{e}-009$ & $1.1369 \mathrm{e}-013$ & $8.4937 \mathrm{e}-007$ & $2.9104 \mathrm{e}-011$ \\
\hline 24 & $3.2596 \mathrm{e}-009$ & $5.1159 \mathrm{e}-012$ & $7.4506 \mathrm{e}-007$ & $8.7311 \mathrm{e}-010$ \\
\hline 28 & $5.3551 \mathrm{e}-009$ & $1.1738 \mathrm{e}-010$ & $7.7486 \mathrm{e}-007$ & $4.2987 \mathrm{e}-009$ \\
\hline
\end{tabular}

\subsection{Error Bound for Fractional Order Derivatives}

We deduce the effect of rounding errors on the largest element in the matrix $\mathbf{D}^{(\alpha)}$ in case of at the shifted Chebyshev extreme points by using Eq. (14), i.e.,

$$
d_{01}^{(0.5)}=\frac{2}{N} \sum_{n=0}^{N} 2(-1)^{n} \theta_{n}\left(2 x_{n}-1\right) n^{2}\left(1+(-1)^{n}\right)
$$

with the error bound

$$
\begin{aligned}
& \tilde{d}_{01}^{(0.5)}-d_{01}^{(0.5)}=\frac{4}{N} \sum_{n=0}^{N} 2(-1)^{n} \theta_{n} n^{2}\left[1+(-1)^{n}\right] \delta_{n} \\
& \leq\left[\frac{-8}{3} N^{2}+4 N+(-1)^{N}(1+N)+\frac{4}{3}\right] \delta
\end{aligned}
$$

The error in the element $d_{01}^{(0.5)}$ is of order $O\left(N^{2} \delta\right)$. Table (4.3) shows the error bound.

Table (4.3): Computed error $\tilde{d}_{01}^{(1)}-d_{01}^{(1)}$

\begin{tabular}{|c|c|}
\hline $\mathrm{N}$ & Eq. $(167$ \\
\hline 8 & $4.7286 \mathrm{e}-014$ \\
\hline 12 & $9.9086 \mathrm{e}-014$ \\
\hline 16 & $1.6983 \mathrm{e}-013$ \\
\hline 20 & $2.5952 \mathrm{e}-013$ \\
\hline 24 & $3.6815 \mathrm{e}-013$ \\
\hline 28 & $4.9573 \mathrm{e}-013$ \\
\hline
\end{tabular}

Some numerical results with $\alpha=0.2,0.4$ for the test function $f(x)=x^{8}$ compared with its exact fractional derivatives are shown in Figures (4.1) and (4.2), respectively.

The examples below show that it is best to compute the fractional differentiation matrix accurately instead of classical matrix. In additional, the well known the negative sum trick can be applied to the final matrix. The penalty for ignoring this advice is not always severe. It is most important to compute 


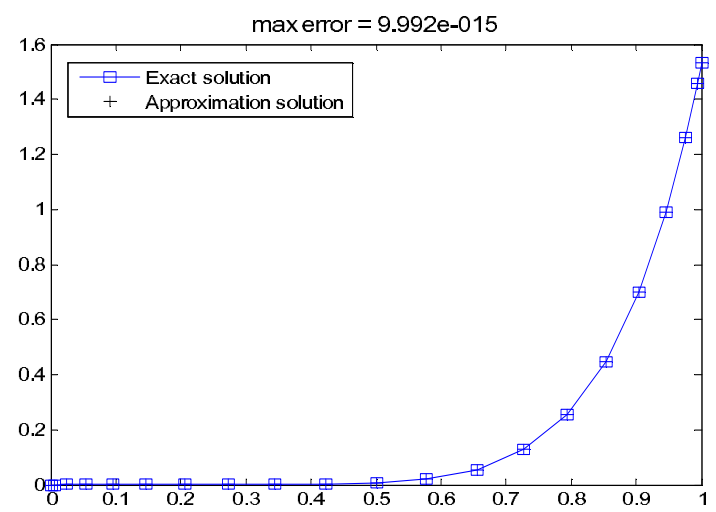

Fig(4.1) Fractional derivative of $\mathrm{f}(\mathrm{x})=\mathrm{x}^{8}$ for $\mathrm{N}=20$ at $\alpha=0.2$

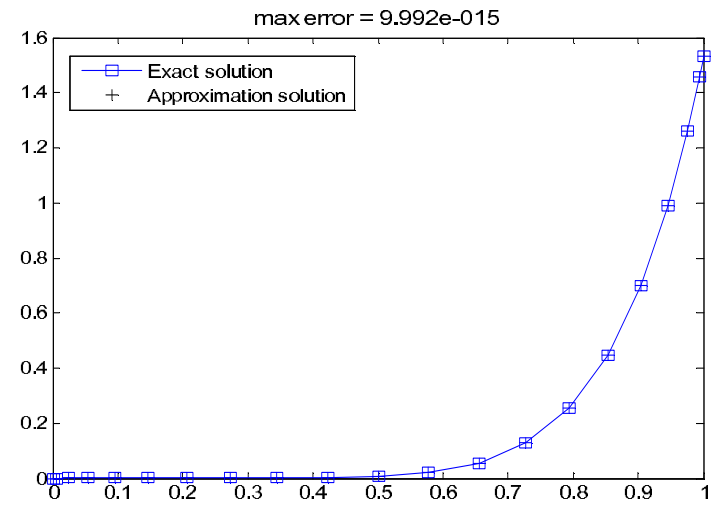

Fig(4.2) Fractional derivative of $\mathbf{f}(\mathbf{x})=\mathbf{x}^{8}$ for $\mathrm{N}=20$ at $\alpha=0.4$

the first few and last few rows of $\mathbf{D}^{(\alpha)}$ accurately. Because of boundary conditions, these are precisely the rows of $\mathbf{D}^{(\alpha)}$ which often are removed from the fractional differentiation matrix.

\section{Application}

We now consider some of numerical examples to illustrate the comparative performance of the fractional differentiation matrix given in Eq. (14).

Example 1. Consider the following fractional differentiation equation:

$$
D^{\alpha} y(x)+y(x)=\frac{2}{\Gamma(3-\alpha)} x^{2-\alpha}-\frac{1}{\Gamma(2-\alpha)} x^{1-\alpha}=+x^{2}-x,
$$

with initial conditions $y(0)=0$, which has exact solution $y(x)=x^{2}-x$. The 
following figures (4.3), (4.4), (4.5), and (4.6) show the approximate solutions compared with the exact solution by using Eq. (14) at $\alpha=0.3,0.5,0.7,0.9$, respectively and $\mathrm{N}=20$.

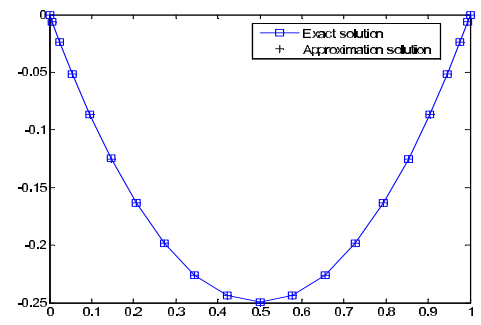

Fig. (4.3): $\alpha=0.3$

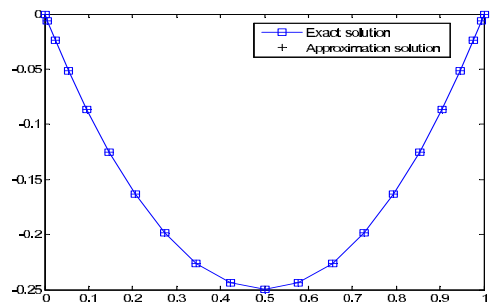

Fig. (4.5): $\alpha=0.7$

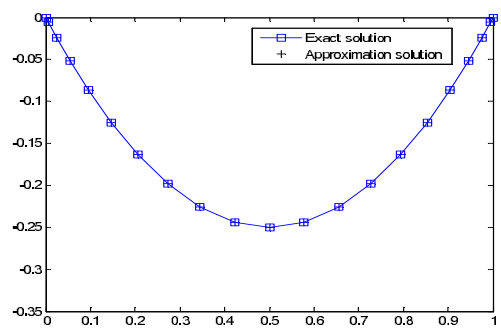

Fig. (4.4): $\alpha=0.5$

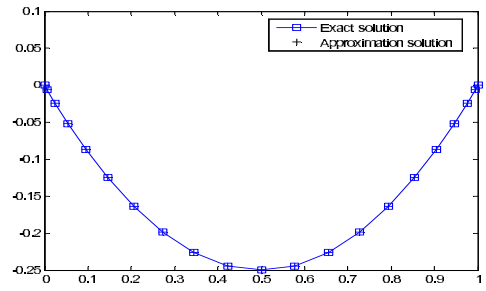

Fig. (4.6): $\alpha=0.9$

Table (4.4):

\begin{tabular}{|c|c|c|c|c|c|}
\hline$\alpha$ & $\mathrm{N}=8$ & $\mathrm{~N}=12$ & $\mathrm{~N}=16$ & $\mathrm{~N}=20$ & $\mathrm{~N}=24$ \\
\hline 0.3 & $1.6653 \mathrm{e}-16$ & $3.6082 \mathrm{e}-16$ & $4.7184 \mathrm{e}-16$ & $8.0491 \mathrm{e}-16$ & $2.5846 \mathrm{e}-13$ \\
\hline 0.5 & $2.2204 \mathrm{e}-16$ & $1.9429 \mathrm{e}-16$ & $4.9266 \mathrm{e}-16$ & $9.7145 \mathrm{e}-16$ & $7.5495 \mathrm{e}-15$ \\
\hline 0.7 & $6.9389 \mathrm{e}-16$ & $6.9389 \mathrm{e}-16$ & $1.2370 \mathrm{e}-15$ & $9.7145 \mathrm{e}-16$ & $1.2455 \mathrm{e}-15$ \\
\hline 0.9 & $2.4286 \mathrm{e}-16$ & $9.7838 \mathrm{e}-16$ & $4.9960 \mathrm{e}-16$ & $3.6082 \mathrm{e}-16$ & $2.0609 \mathrm{e}-15$ \\
\hline
\end{tabular}

Example 2. Consider the following fractional differential equation:

$$
D^{2} y(x)+D^{3 / 4} y(x)+y(x)=x^{2}+6 x+\frac{8.533333333}{\Gamma(0.25)} x^{2.25}
$$

with initial conditions $y(0)=y^{\prime}(0)=0$ which has exact solution $y(x)=x^{3}$. The maximum absolute error is shown in Table (4.5) for different numbers of shifted CGL points compared with the method [4].

Table (4.5) : 


\begin{tabular}{|c|c|c|}
\hline $\mathrm{N}$ & Ref.[4] & Eq.(14) \\
\hline 8 & $1.91-07$ & $5.74 \mathrm{e}-12$ \\
\hline 12 & $1.52 \mathrm{e}-08$ & $5.75 \mathrm{e}-12$ \\
\hline 16 & $2.52 \mathrm{e}-09$ & $5.75 \mathrm{e}-12$ \\
\hline 20 & $6.22 \mathrm{e}-10$ & $5.69 \mathrm{e}-12$ \\
\hline 24 & $1.96 \mathrm{e}-10$ & $5.92 \mathrm{e}-12$ \\
\hline
\end{tabular}

Example 3. Consider the following nonlinear fractional differential equation

$$
D^{\alpha} y(x)=1+y(x)^{2}, n-1<\alpha \leq n
$$

with initial conditions $y^{(i)}(0)=0, i=0,1, \ldots, n-1$.

The exact solution of Eq. (20) for $\alpha=1$ is $y(x)=\tan (x)$. The following figure (4.7) shows the comparison of $y(x)$ for $\mathrm{N}=20$ and $\alpha=0.9,0.95,1,1.5,2.5$

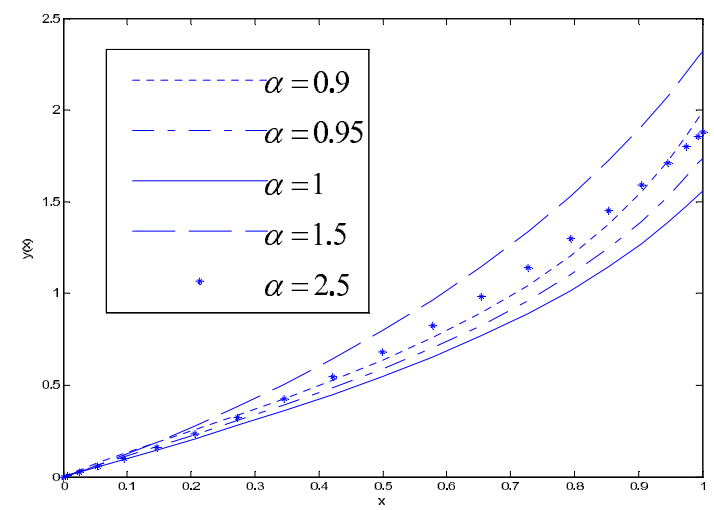

Fig(4.7) Comparison of the approximate solution for example (3)

Example 4. Consider the following nonlinear fractional differential equation:

$$
D^{\alpha} y(x)+y(x)^{2}=1,0<\alpha \leq 1
$$

subject to the initial condition $y(0)=0$. The exact solution when $\alpha=1$ is

$$
y(x)=\frac{e^{2 x}-1}{e^{2 x}+1} .
$$

In the case of $\alpha=0.5 .0 .7,0.9,1$, The approximation solution obtained by the present matrix at $\mathrm{N}=20$ is shown in Fig. (4.8) to make it easier to compare with the analytic solution 


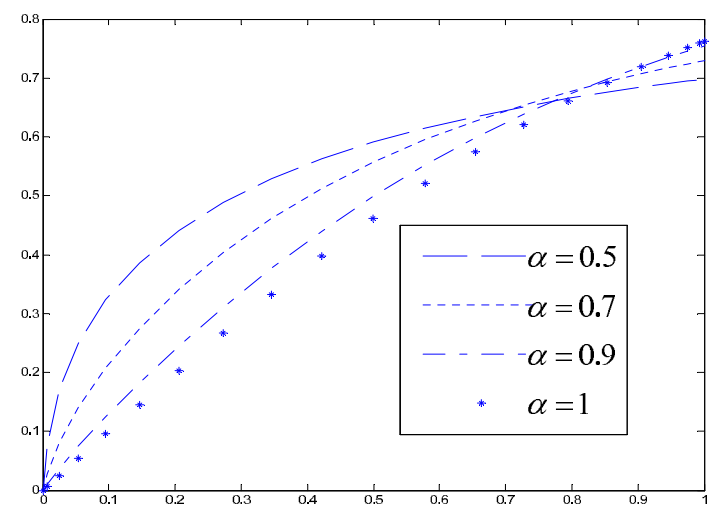

Fig(4.8) Copmarison of the approximate solution for example (4)

\section{Conclusion}

In this paper, the fundamental goal has been constructed by new fractional differentiation matrices. We proposed a numerical algorithm to solve the general nonlinear fractional-order differential equations using CGL points and approximating directly the solution using the shifted Chebyshev polynomials. The goal has been achieved by using the fractional differentiation matrices. The illustrative examples confirm the validity of the new differentiation matrix and it is employed to solve other strongly fractional differential equations. We note that similar techniques can be applied to tau and collocation methods using Legendre polynomials.

\section{References}

[1] C.W. Clenshaw, A.R. Curtis, A method for numerical integration on an automatic computer, Numer. Math., 2 (1960), 197-205.

[2] E.H. Doha, The ultraspherical coefficients of moments of a general order derivative of an infinitely differentiable function, J. Comput. and Appl. Math., 89 (1998), 53-72.

[3] W.S. Don, A. Solomonoff, Accuracy and speed in computing the Chebyshev collocation derivative, SIAM J. Sci. Comput., 16 (1995), 1253-1268.

[4] E.H. Doha, A.H. Bhrawy, S.S. Ezz-Eldien, Efficient Chebyshev spectral methods for solving multi-term fractional orders differential equations, $A p$ plied Mathematical Modelling, 35 (2011), 5662-5672. 
[5] E.M.E. Elbarbary, M. El-Kady, Chebyshev finite difference approximation for the boundary value problems, Appl. Math. and Comput., 139 (2003), 513-523.

[6] E.M.E. Elbarbary, S.M. El-Sayed, Higher-order pseudospectral differentiation matrices, Appl. Numer. Math., 55 (2005), 425-438.

[7] J.C. Mason, D.C. Handscomb, Chebyshev Polynomials, Chapman and Hall CRC Press Company (2002).

[8] Karageorghis, A note on the Chebyshev coefficients of the general-order derivative of an infinitely differentiable function, Comput. Appl. Math., 21 (1988), 383-386.

[9] R. Baltensperger, M.R. Trummer, Spectral differencing with a twist, SIAM J. Sci. Comput., 24 (2003), 1465-1487.

[10] R. Baltensperger, Improving the accuracy of the matrix differentiation method for arbitrary collocation points, Appl. Numer. Math., 33 (2000), 143-149. 
